\title{
Simultaneous demultiplexing, data regeneration, and clock recovery with a single semiconductor optical amplifier-based nonlinear-optical loop mirror
}

\author{
I. D. Phillips, A. Gloag, P. N. Kean, N. J. Doran, and I. Bennion \\ Photonics Research Group, Department of Electronic Engineering and Applied Physics, Aston University, Aston Triangle, \\ Birmingham B47ET, UK \\ A. D. Ellis \\ Photonic and Quantum Processing, BT Laboratories, Martlesham Heath, Ipswich, Suffolk IP5 7RE, England
}

Received April 15, 1997

\begin{abstract}
We demonstrate simultaneous demultiplexing, data regeneration and clock recovery at $10 \mathrm{Gbits} / \mathrm{s}$, using a single semiconductor optical amplifier-based nonlinear-optical loop mirror in a phase-locked loop configuration. (C) 1997 Optical Society of America
\end{abstract}

In periodically amplified return-to-zero transmission systems, signal-spontaneous beat noise from each amplifier will induce random frequency fluctuations, which are subsequently translated into a timing jitter. ${ }^{1}$ This jitter, combined with amplitude fluctuations, will limit the maximum transmission distance. Electronic signal regenerators can be used but will inevitably introduce a bandwidth limitation into a transmission system (the so-called electronic bottleneck). To overcome this problem the next generation of high-speed optical time-division multiplexed networks will rely on all-optical signal processing.

A typical network architecture will consist of a number of network nodes. It is recognized that each node will need to perform basic functions including demultiplexing, ${ }^{2}$ add-drop multiplexing, and clock recovery. ${ }^{3,4}$ Data regeneration and wavelength conversion may also be required at certain nodes on a large network, and a great deal of research has been conducted on devices that will perform these functions. ${ }^{5,6}$ Conventionally each process is performed independently. In this Letter we demonstrate experimentally simultaneous clock recovery, demultiplexing, and data regeneration in a single semiconductor optical amplifier-based nonlinear-optical loop mirror (SOA-NOLM). ${ }^{7}$

In a conventional (two-wavelength) switched nonlinear-optical loop mirror (NOLM) an intense control pulse induces a nonlinear phase difference between counterpropagating signals through cross-phase modulation. The SOA-NOLM is different from the NOLM in that the nonlinearity is provided by an induced change in optical properties of the semiconductor optical amplifier (SOA) provided by a saturating control pulse. Situating the SOA slightly off center causes counterpropagating signals to probe the device at different times and thus to acquire a phase differential that switches the loop. The width of the switching window is therefore controlled by the offset from the center of the loop. The various advantages of the SOA-NOLM compared with the standard fiber NOLM include low switching power, compact size, and stability.

Figure 1 shows the experimental setup. Clock recovery is achieved by use of the optical sampling properties of the loop mirror ${ }^{8}$ as a phase-sensitive detector, allowing low-speed feedback electronics to control the locally generated optical clock.

One can achieve data regeneration with a fiber NOLM by letting the degraded input data stream switch the interferometer. ${ }^{9}$ To achieve this result in a SOA-NOLM without patterning, we must meet two criteria. First, the SOA must be situated within half a clock period of the loop center, to ensure that the corresponding components of the same signal pulse experience the phase evolution induced by a single data (control) pulse. ${ }^{10}$ Second, we require the gain to recover completely between pulses. Although switching of a SOA device has been achieved faster

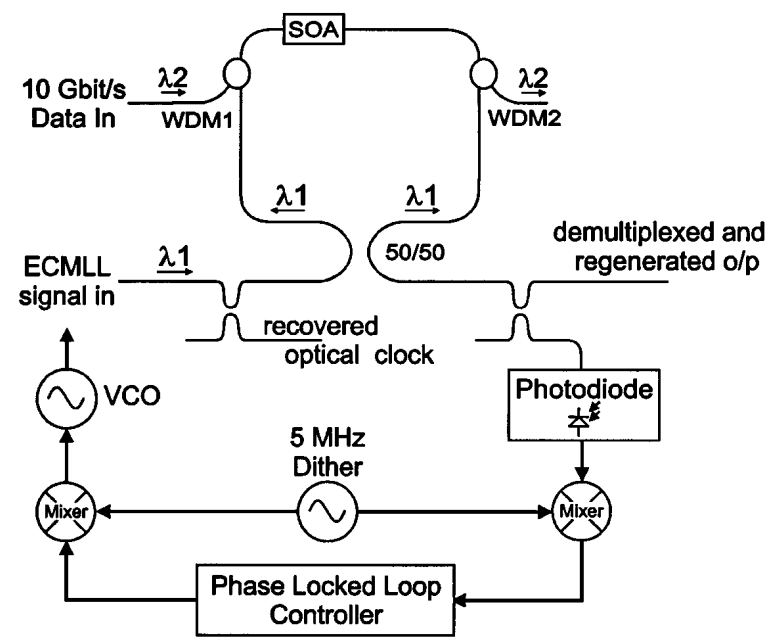

Fig. 1. Experimental setup for simultaneous demultiplexing, data regeneration, and clock recovery. WDM's, wavelength division multiplexers; o/p, output; VCO, voltage-controlled oscillator. 
than the gain recovery time, ${ }^{11,12}$ it was done with a regular control pulse train in an architecture that prevented regeneration.

We constructed the SOA-NOLM by using a $1000-\mu \mathrm{m}$ multi-quantum-well semiconductor optical amplifier, a variable fiber stretcher to control the switching window ( $\approx 35 \mathrm{ps})$, and a pair of $1535 / 1555 \mathrm{~nm}$ wavelengthdivision multiplexers to inject and extract the control signal from the loop. Polarization controllers were included to bias the loop into either transmission or reflection. The recovery time of the SOA was $100 \mathrm{ps,}$ permitting a switching bandwidth of $10 \mathrm{GHz}$. A local optical clock was generated by an external-cavity modelocked laser (ECMLL) running at $1533 \mathrm{~nm}$. When modulated at $2.5 \mathrm{GHz}$, the ECMLL produced 20-ps pulses with a 1.5-ps timing jitter. At $10 \mathrm{GHz}$ the pulse duration was 15 ps with a 1.2-ps jitter.

To demonstrate data regeneration, we switched a $10-\mathrm{GHz}$ pulse train from the ECMLL by using a degraded 10-Gbit/s pseudorandom data stream, which we generated by modulating the output from an actively mode-locked fiber ring laser. This laser and the ECMLL were both triggered from the same signal generator, ensuring a fixed phase relationship. Figure 2 shows the input data with a $Q$ of 6.4 [trace (a)] and the regenerated output data with a $Q$ of 10.3 [trace (b)]. By modulating the ECMLL at $2.5 \mathrm{GHz}$ (phase locked to the data source) we achieved all-optical 102.5-Gbit/s demultiplexing with inherent data regeneration; see Fig. 3. The 10-Gbit/s input data stream was degraded to a $Q$ of 7.0 and a timing jitter of $6.0 \mathrm{ps}$. The demultiplexed and regenerated output had a $Q$ of 13.9 and a timing jitter of $1.5 \mathrm{ps.} \mathrm{This} \mathrm{re-}$ sult shows the excellent regeneration properties of the SOA-NOLM.

For demultiplexing applications it is essential to maintain a good extinction ratio to ensure that unwanted channels are completely removed. In this case interchannel cross talk is eliminated simply because of the absence of light from the clock pulses that are used to probe the switching of the SOA-NOLM.

Because the output from the SOA-NOLM represents a convolution of the two input pulses (signal and control), the time-averaged intensity output will vary as the phase between the two pulse trains varies. This inherent phase-sensing property of the loop permits its use as an all-optical phase detector analogous to a mixer in a classical electronic phase-locked loop. We can thus achieve clock recovery by ensuring that the output intensity from the loop is kept at a maximum. As the phase wanders from optimum, the total throughput of the loop will drop, as pulses are not switched completely. To achieve $100 \%$ switching from the incoming data stream it is therefore necessary to impose a small dither onto the locally produced clock, permitting locking to the intensity differential by use of the extracted dither component as an error signal. ${ }^{13}$

A-5 $\mathrm{MHz}$ frequency dither was applied to the optical clock via the voltage-controlled oscillator used to drive the ECMLL. With a small proportion of the output monitored on a $125-\mathrm{MHz}$ photodiode, the dither signal at $5 \mathrm{MHz}$ was extracted by a narrow-band filter and a mixer. The resulting signal, representing the phase error between the two pulse streams, was then fed to the standard phase-locked loop control electronics used to drive the voltage-controlled oscillator. The setup can be seen schematically in the lower half of Fig. 1. In this way a closed loop was formed, with any fluctuations in phase being constantly corrected for.

Figure $4 \mathrm{a}$ shows the degraded 10-Gbit/s input data stream with a $Q$ of 7.22 ; Fig. $4 \mathrm{~b}$ shows the 10-2.5-Gbit/s demultiplexed and regenerated data stream with a $Q$ of 15.66 ; Fig. 4c shows the recovered $2.5-\mathrm{GHz}$ optical clock with timing jitter of only $1.5 \mathrm{ps}$.

Figure 5 shows the microwave spectrum of the recovered electrical clock. At a span of $200 \mathrm{kHz}$ the noise pedestal is $35 \mathrm{~dB}$ down. By increasing the span to $10 \mathrm{MHz}$ we can see the sidebands from the frequency

(a)

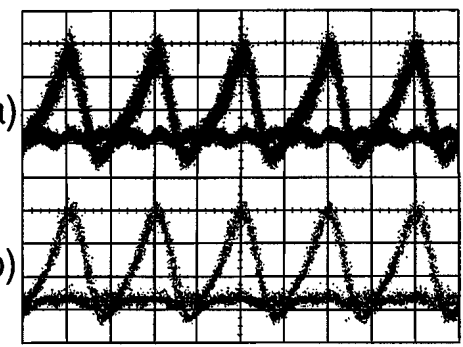

Fig. 2. All-optical 10-Gbit/s data regeneration: (a) 10 -Gbit/s degraded input data stream, $Q=6.4$; (b) $10-\mathrm{Gbit} / \mathrm{s}$ regenerated data stream, $Q=10.3$.

(a)

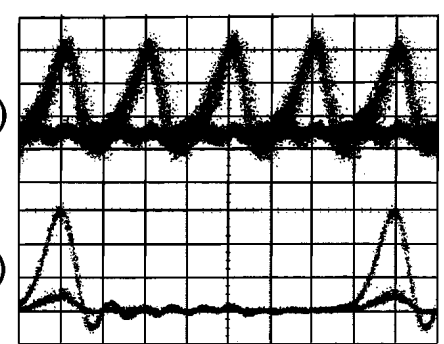

Fig. 3. All-optical 10-2.5-Gbit/s demultiplexing and data regeneration: (a) $10-\mathrm{Gbit} / \mathrm{s}$ input data stream, $Q=7.0$, timing jitter $6.0 \mathrm{ps}$; (b) 2.5-Gbit/s demultiplexed and regenerated data stream, $Q=13.9$, timing jitter $1.5 \mathrm{ps}$.

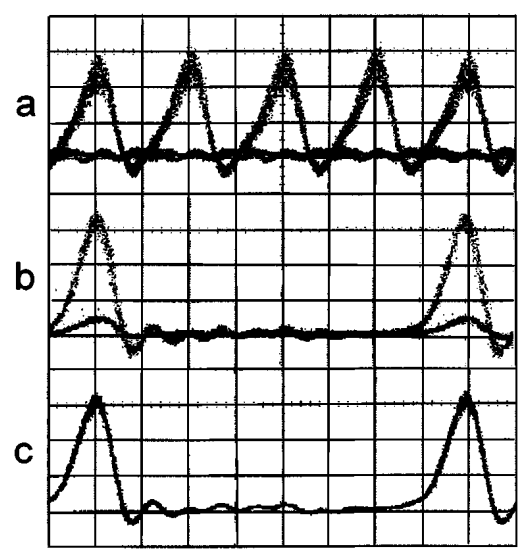

Fig. 4. Simultaneous demultiplexing, data regeneration, and clock recovery: a, 10-Gbit/s degraded input data stream, $Q=7.22 ; \mathrm{b}, 2.5$-Gbit/s demultiplexed and regenerated data stream, $Q=15.66 ; \mathrm{c}, 2.5-\mathrm{GHz}$ recovered clock, timing jitter $1.5 \mathrm{ps}$. 

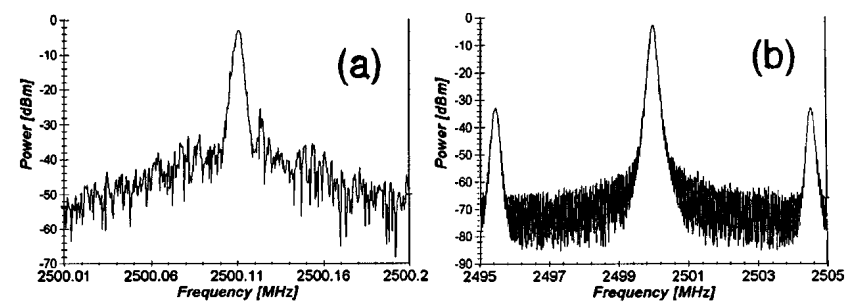

Fig. 5. Microwave spectrum of the recovered electrical clock: (a) span $200 \mathrm{kHz}$, noise pedestal $35 \mathrm{~dB}$ down; (b) span $10 \mathrm{MHz}$; dither components at $\pm 5 \mathrm{MHz}$ $30 \mathrm{~dB}$ down.

modulation of the signal used to achieve optimum signal regeneration; these are $30 \mathrm{~dB}$ down and cause a frequency-modulated timing jitter of $6 \mathrm{ps}$ on the recovered clock. We can eliminate this jitter when making measurements by ensuring that the optical and the electrical clocks are in phase.

We have shown, for the first time to our knowledge, simultaneous demultiplexing, data regeneration, and clock recovery from 10 to $2.5 \mathrm{Gbit} / \mathrm{s}$, using a single SOA-NOLM. We combined the SOA-NOLM's demultiplexing properties with its inherent sampling ability. We are currently investigating the performance of this device for simultaneous clock recovery and data regeneration at $10 \mathrm{Gbits} / \mathrm{s}$. The recovery time of the device used in this research limits switching speeds to $\sim 10 \mathrm{GHz}$. By trading off nonlinearity and switching energy to achieve a faster recovery time, and by the use of new SOA design and fabrication methods (e.g., low-temperature-grown strained multiplequantum-well InGaAs/InAlAs doped with beryllium ${ }^{14}$ ), we should be able to realize a recovery time of $\sim 1 \mathrm{ps}$, permitting switching and regeneration of data at a rate of as much as $100 \mathrm{Gbits} / \mathrm{s}$.
The authors gratefully acknowledge financial support from the Engineering and Physical Sciences Research Council (UK) and a Cooperative Award in Science and Engineering studentship from BT Photonic and Quantum Processing.

\section{References}

1. J. P. Gordon and H. A. Haus, Opt. Lett. 11, 665 (1986).

2. D. M. Patrick and R. J. Manning, Electron. Lett. 30, 151 (1994).

3. E. Jahn, N. Agrawal, M. Arbert, H. J. Ehrke, D. Franke, R. Ludwig, W. Pieper, H. G. Weber, and C. M. Weinert, Electron. Lett. 31, 1857 (1995).

4. S. Kawanishi and M. Saruwatari, Electron. Lett. 24, 1452 (1988).

5. J. K. Lucek and K. Smith, Opt. Lett. 18, 1226 (1993).

6. I. D. Phillips, A. Gloag, N. J. Doran, I. Bennion, and A. D. Ellis, in Optical Fiber Communication Conference, Vol. 7 of 1997 OSA Technical Digest Series (Optical Society of America, Washington, D.C., 1997), p. 273.

7. A. D. Ellis, D. M. Patrick, D. Flannery, R. J. Manning, D. A. O. Davies, and D. M. Spirit, J. Lightwave Technol. 13, 761 (1995).

8. B. P. Nelson and N. J. Doran, Electron. Lett. 27, 204 (1991).

9. M. Jinno and M. Abe, Electron. Lett. 28, 1350 (1992).

10. D. A. O. Davies, A. D. Ellis, and G. Sherlock, Electron. Lett. 31, 1000 (1995).

11. A. D. Ellis and D. M. Spirit, Electron. Lett. 29, 2115 (1993).

12. K. Suzuki, K. Iwatsuki, S. Nishi, and M. Saruwatari, Electron. Lett. 30, 1501 (1994).

13. H. Bülow, Electron. Lett. 31, 1937 (1995).

14. R. Takahashi, Y. Kawamura, and H. Iwamura, Appl. Phys. Lett. 68, 153 (1996). 